\title{
Effects of edaravone, a free-radical scavenger, on bleomycin-induced lung
} injury in mice

\author{
S. Tajima*,\#, M. Bando*, Y. Ishii*, T. Hosono*, H. Yamasawa*, S. Ohno*, T. Takada\#,

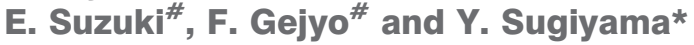

ABSTRACT: Reactive oxygen species play an important role in the pathogenesis of acute lung injury and pulmonary fibrosis. The present authors hypothesise that edaravone, a free-radical scavenger, is able to attenuate bleomycin (BLM)-induced lung injury in mice by decreasing oxidative stress.

Lung injury was induced in female ICR mice by intratracheal instillation of $5 \mathrm{mg} \cdot \mathrm{kg}^{-1}$ of BLM. Edaravone $\left(300 \mathrm{mg} \cdot \mathrm{kg}^{-1}\right)$ was administered by intraperitoneal administration $1 \mathrm{~h}$ before BLM challenge.

Edaravone significantly improved the survival rate of mice treated with BLM from 25 to $90 \%$, reduced the number of total cells and neutrophils in bronchoalveolar lavage fluid (BALF) on day 7 , and attenuated the concentrations of lipid hydroperoxide in BALF and serum on day 2. The fibrotic change in the lung on day $\mathbf{2 8}$ was ameliorated by edaravone, as evaluated by histological examination and measurement of hydroxyproline contents. In addition, edaravone significantly increased the prostaglandin $\mathrm{E}_{2}$ concentration in BALF on day 2.

In summary, edaravone was shown to inhibit lung injury and fibrosis via the repression of lipid hydroperoxide production and the elevation of prostaglandin $E_{2}$ production in the present experimental murine system.

KEYWORDS: Bleomycin, edaravone, free-radical scavenger, lung injury, pulmonary fibrosis

diopathic pulmonary fibrosis (IPF) is defined as a specific form of chronic fibrosing inter-

stitial pneumonia limited to the lung [1]. The aetiology of IPF is not known, and IPF remains a devastating disease with a 5 -yr mortality rate of $>50 \%$ [1]. Unfortunately, the pathogenesis of IPF is also incompletely understood. Although several drugs have been used or tested for IPF, there is no established treatment that definitely improves its outcome [1]. Thus, new therapies are awaited, based on new understanding of the pathogenesis of IPF. There is considerable evidence that oxygengenerated free radicals play a major role in inflammatory and immune-mediated tissue injury [2-4]. DEMEDTs et al. [5] have shown that acetylcysteine, a precursor of the major antioxidant glutathione, administered at a daily dose of $1,800 \mathrm{mg}$ in combination with prednisone and azathioprine, preserves vital capacity and carbon monoxide diffusing capacity better in patients with IPF than the combination of prednisone and azathioprine alone. These findings suggest that an oxidant-antioxidant imbalance may contribute to the disease process in IPF.
Bleomycin (BLM), an antineoplastic agent, induces pulmonary fibrosis as an adverse effect, since the hydrolase that inactivates BLM is relatively scarce in lung tissue. The mechanism of the antineoplastic effect of BLM is that the BLM-iron complex reduces molecular oxygen to superoxide and hydroxy radicals that can then attack DNA and cause strand cleavage [6]. The role of oxygen free radicals has been supported by studies showing that the addition of superoxide dismutase, an oxygen free-radical scavenger, inhibited BLM-induced DNA breakage and cellular damage in vitro [7-10]. Therefore, a BLMinduced pulmonary fibrosis model in mice is a helpful tool to examine the general mechanism of fibrosis, especially the mechanism mediated by oxygen free radicals.

Edaravone (3-methyl-1-phenyl-2-pyrazolin-5-one) is a potent free-radical scavenger and has the antioxidant ability to inhibit lipid peroxidation [11]. Therefore, it is speculated that edaravone administration might ameliorate the tissue damage induced by reactive oxygen species (ROS).
AFFILIATIONS

*Division of Pulmonary Medicine, Dept of Medicine, Jichi Medical University, Shimotsuke, and \#Division of Respiratory Medicine, Niigata University Graduate School of Medical and Dental Sciences, Niigata, Japan.

CORRESPONDENCE

S. Tajima

Division of Respiratory Medicine Niigata University Graduate School of Medical and Dental Sciences 1-757 Asahimachi-dori Chuo-ku

Niigata 951-8510 Japan Fax: 81252270775 E-mail: tajimash@ med.niigata-u.ac.jp

Received:

December 062007 Accepted after revision: July 022008

SUPPORT STATEMENT

This study was supported by the Health and Labour Sciences Research Grants on Diffuse Lung Diseases from the Japanese Ministry of Health, Labour and Welfare.

STATEMENT OF INTEREST A statement of interest for this study can be found at www.erj.ersjournals.com/misc/ statements.shtml 
Edaravone has protective effects on both hemispheric embolisation and transient cerebral ischaemia, and has, therefore, been used clinically to treat acute brain infarction in Japan [12-14]. ITO et al. [15] have shown that edaravone ameliorated the lung injury induced by intestinal ischaemia/reperfusion. In the study by ITO et al. [15], edaravone decreased the neutrophil infiltration, the lipid membrane peroxidation and the expression of interleukin (IL)-6 mRNA in the lungs, resulting in a reduction in mortality. Most recently, ASAI et al. [16] have shown that edaravone suppressed BLM-induced acute pulmonary injury in rabbits. They reported that a 10-day intravenous edaravone administration beginning 3 days prior to intratracheal instillation of BLM significantly attenuated the acute BLM-induced lung injury and the numbers of both terminal deoxynucleotidyltransferasemediated deoxyuridine triphosphate-positive (apoptotic) and transforming growth factor- $\beta$ positive cells on day 7 [16]. Although the results of AsAI et al. [16] support the present authors' hypothesis, it was thought that several critical points were lacking, as follows: 1) collagen accumulation at the late fibrosing stage was not evaluated; and 2) bronchoalveolar lavage (BAL) was not performed and ROS was not measured in order to evaluate inhibitory effects on the inflammatory process. Accordingly, in the present study, a BLM-induced pulmonary fibrosis model was used in mice, which is a more common animal lung fibrosis model than the rabbit model used by AsAi et al. [16], to investigate the ability of edaravone to: 1) inhibit pulmonary fibrosis; or 2) decrease lung inflammation and attenuate ROS.

\section{MATERIALS AND METHODS}

\section{Mice, cells and reagents}

All mice received humane care in accordance with the Guide for the Care and Use of Laboratory Animals, US National Institutes of Health (Bethesda, MD, USA). The study protocol was approved by the Ethics Committee of Jichi Medical University (Tochigi, Japan). Female ICR mice, 6-8 weeks of age, were obtained from Japan SLC (Tochigi, Japan) and housed in the animal facility of Jichi Medical University. BLM was purchased from Nippon Kayaku (Tokyo, Japan). Edaravone was a gift from Mitsubishi Pharma Corporation (Tokyo, Japan). It was dissolved in a small amount of $1 \mathrm{~N} \mathrm{NaOH}$ solution, the $\mathrm{pH}$ was adjusted to 7.0 with $1 \mathrm{~N} \mathrm{HCl}$ and the concentration was adjusted to $3 \mathrm{mg} \cdot \mathrm{mL}^{-1}$ in the saline solution.

\section{BLM-induced pulmonary fibrosis model}

To induce pulmonary fibrosis, ICR mice were treated with intratracheal BLM on day 0. The ICR mice were anaesthetised by the intraperitoneal administration of $0.01 \mathrm{~mL} \cdot \mathrm{g}^{-1}$ of $10 \%$ pentobarbital sodium solution (Abbott Laboratories, North Chicago, IL, USA), followed by intratracheal instillation of $5 \mathrm{mg} \cdot \mathrm{kg}^{-1}$ body weight of BLM in $50 \mu \mathrm{L}$ of sterile isotonic saline. The control animals received intratracheal saline only. Edaravone dissolved in saline or the same volume of saline was administered by a single intraperitoneal injection either $1 \mathrm{~h}$ before or $24 \mathrm{~h}$ after BLM injection. To ascertain the optimal dose of edaravone for the proposed experiment, mice were given edaravone at a dose of $0,3,30$ or $300 \mathrm{mg} \cdot \mathrm{kg}^{-1}$ or the same volume of saline (10-12 mice in each group). The mice were killed under anaesthesia on day 2, 7 or 28 after BLM instillation, for examination. On day 28 , the left lung lobes were used for hydroxyproline assay. In the mice receiving pre-administration of $300 \mathrm{mg} \cdot \mathrm{kg}^{-1}$ edaravone with BLM instillation, BAL was performed on days 2 and 7. In addition, histological examination was performed on day 28. The present authors randomly selected six or 10 mice samples from each group. Mortality calcuation, hydroxyproline assay, histological examination and BAL analysis were performed independently.

\section{Sampling of BAL fluid and serum}

Under anaesthesia, as previously described, blood samples were obtained from the right atrium at each time-point. After centrifugation at $3,000 \times g$ for $10 \mathrm{~min}$ at $4^{\circ} \mathrm{C}$, the serum was frozen and stored at $-80^{\circ} \mathrm{C}$ until it was assayed. BAL was performed four times through a tracheal cannula with $0.7 \mathrm{~mL}$ of saline. In each mouse examined, $\sim 2.5 \mathrm{~mL}(90 \%)$ of BAL fluid (BALF) was recovered. A $100-\mu \mathrm{L}$ aliquot was used for the total cell count, and the remainder was immediately centrifuged at $1,000 \times g$ for $10 \mathrm{~min}$. The total cell count was prepared using a haemocytometer, and cell differentiation was determined for $>500$ cells on cytocentrifuge slides with WrightGiemsa staining. The supernatants of BALF were stored at $80^{\circ} \mathrm{C}$ until used.

\section{Morphological evaluation}

Histopathological evaluation was performed on day 28 in the BLM-induced pulmonary fibrosis model. Both lungs were removed and inflated with $10 \%$ formaldehyde neutral buffer solution, and longitudinal tissue sections were stained with haematoxylin and eosin.

\section{Assay of hydroxyproline}

Hydroxyproline in the murine lung on day 28 after BLM instillation was assayed according to the commonly used

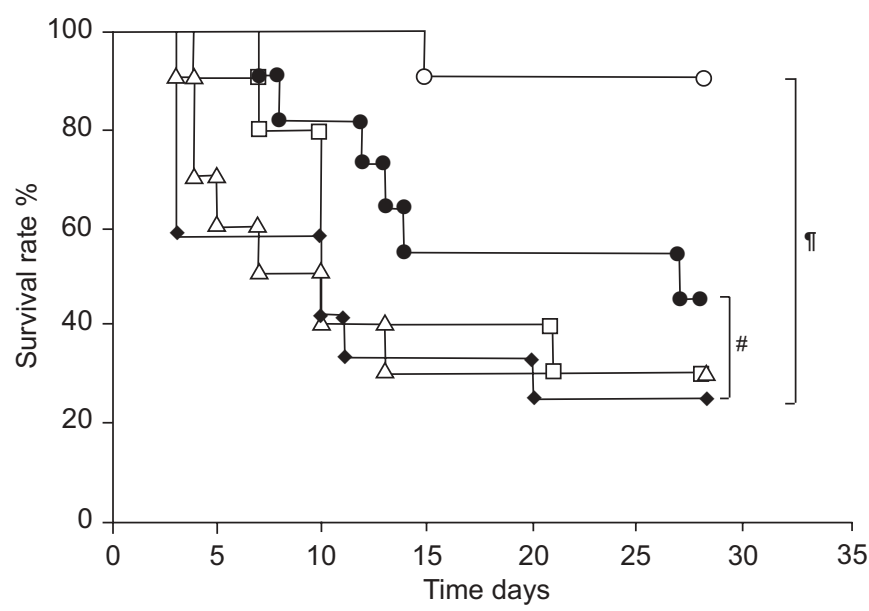

FIGURE 1. Effects of edaravone on mortality in a bleomycin (BLM)-induced lung injury mouse model. The survival rates of five study groups of mice are shown over a 28-day observation period (10-12 mice in each group). The four BLM + edaravone groups received single intraperitoneal infusion of edaravone as follows. $\bigcirc$ : high dose of edaravone (pre-treatment, $300 \mathrm{mg} \cdot \mathrm{kg}^{-1}$ ); $\square$ : intermediate dose of edaravone (pre-treatment, $30 \mathrm{mg} \cdot \mathrm{kg}^{-1}$ ); $\triangle$ : low dose of edaravone (pre-treatment, $3 \mathrm{mg} \cdot \mathrm{kg}^{-1}$ ) administered as a single intraperitoneal infusion $1 \mathrm{~h}$ before the instillation of BLM; $\bullet$ : high dose of edaravone as a single intraperitoneal infusion $24 \mathrm{~h}$ after the instillation of BLM (treatment, $300 \mathrm{mg} \cdot \mathrm{kg}^{-1}$ ). The survival rate of the high-dose edaravone group (pre-treatment, $300 \mathrm{mg} \cdot \mathrm{kg}^{-1}$; O) was significantly higher than the group administered intratracheal instillation of BLM $(\bullet ; p<0.05)$ The results for the control group are not shown. ${ }^{*}: p=0.15 ;{ }^{\natural}: p=0.002$ 
procedure of colorimetric measurement (Mitsubishi Kagaku Bio-Clinical Laboratories, Inc., Tokyo) [17, 18]. Hydroxyproline content $\left(\mu \mathrm{g} \cdot \mathrm{lung}^{-1}\right)$ was measured in the left lung of each subject.

\section{Assays for lipid hydroperoxide and prostaglandin $E_{2}$}

The concentrations of lipid hydroperoxide (LPO) in serum and BALF were measured as an indicator of oxidative stress using a Lipid Hydroperoxide Assay kit (Cayman Chemical, Ann Arbor, MI, USA). Prostaglandin (PG) $\mathrm{E}_{2}$ in BALF was quantified using specific immunoassays (Cayman Chemical).

\section{Statistical analysis}

Survival curves were estimated by the Kaplan-Meier method. Comparisons of all curves were carried out using the twotailed log-rank test. Data were expressed as the mean \pm SEM. For multiple comparisons, ANOVA was performed followed by the Fisher's protected least-significant differences method as a post hoc test. Differences between two variables were assessed with the Mann-Whitney U-test. A p-value $<0.05$ was considered to indicate statistical significance.

\section{RESULTS}

Edaravone caused a significant reduction in the mortality of mice with BLM-induced pulmonary fibrosis

The severe lung injury caused by BLM administration was associated with high mortality. To assess the protective effects of edaravone, the compound was injected intraperitoneally in various doses at various times either before or after the BLM instillation. The survival rate of each group is shown in figure 1. In total, nine (75\%) out of 12 animals died from day 3 to 20 after treatment with $5 \mathrm{mg} \cdot \mathrm{kg}^{-1}$ of BLM. However, the pre-administration of $300 \mathrm{mg} \cdot \mathrm{kg}^{-1}$ edaravone significantly improved the survival rate of mice treated with BLM (one out of 10 animals died, $p=0.002$; fig. 1). In contrast, among the mice treated with low-dose edaravone (pre-administration of 3 or $30 \mathrm{mg} \cdot \mathrm{kg}^{-1}$ ) followed by BLM instillation, only three out of 10 mice survived in both dosage groups (fig. 1). The administration of $300 \mathrm{mg} \cdot \mathrm{kg}^{-1}$ edaravone after $24 \mathrm{~h}$ BLM injection (post-treatment administration is the treatment group) did not improve the survival rate of mice treated with BLM (five out of 11 animals died, $\mathrm{p}=0.15$; fig. 1).

\section{Administration of edaravone ameliorated BLM-induced pulmonary fibrosis in mice}

To evaluate the antifibrotic effect of edaravone, mice were treated with $5 \mathrm{mg} \cdot \mathrm{kg}^{-1}$ of BLM and killed on day 28. The fibrotic change in the lung was evaluated by histological examination and measurement of hydroxyproline contents. As shown in figure 2, when $300 \mathrm{mg} \cdot \mathrm{kg}^{-1}$ of edaravone was administered before BLM instillation, a significant reduction of fibrosis in the subpleural areas of the lung was observed. The hydroxyproline assay demonstrated that pre-treatment with edaravone dose-dependently reduced the total hydroxyproline contents in BLM-treated lungs (fig. 3). The posttreatment administration (treatment group) of $300 \mathrm{mg} \cdot \mathrm{kg}^{-1}$ edaravone was also effective in reducing the pulmonary fibrosis caused by BLM.

\section{Analysis of BALF cells in mice with BLM-induced pulmonary fibrosis}

Following this, the cells in BALF were analysed to evaluate the effects of edaravone on the inflammatory responses induced by BLM. Edaravone ( $300 \mathrm{mg} \cdot \mathrm{kg}^{-1}$ body weight) was administered by a single intraperitoneal injection $1 \mathrm{~h}$ before BLM injection. Administration of BLM elevated the number of inflammatory cells, including macrophages, lymphocytes and neutrophils, on days 2 and 7. Pre-administration of edaravone significantly reduced the number of total cells and neutrophils in BALF on day $7(p<0.05$; fig. $4 a$ and $c)$. As shown in figure $4 \mathrm{a}$ and $\mathrm{c}$, the p-value for total cells and neutrophils in BALF between the BLM and BLM + edaravone group were significant but marginal ( $p=0.045$ and $p=0.046$, respectively). Therefore, the present authors did not perform BALF cell analysis or measurement of LPO or $\mathrm{PGE}_{2}$ without pretreatment of $300 \mathrm{mg} \cdot \mathrm{kg}^{-1}$ edaravone.

\section{Effects of edaravone on the amount of LPO in serum and BALF in the BLM model}

One of the possible reasons for the preventive effect of edaravone on BLM-induced lung injury may be its antioxidant effect. To study the antioxidant effect of edaravone, the amount of LPO in the serum and BALF was measured, which is an indicator of oxidative stress [9]. On day 2 after BLM instillation, serum LPO levels were significantly increased compared with those in the control mice ( $p=0.013$; fig. 5a). However, pretreatment with edaravone $\left(300 \mathrm{mg} \cdot \mathrm{kg}^{-1}\right.$ body weight) significantly decreased the levels of LPO in serum, compared with those in the animals treated with BLM alone ( $p=0.001$; fig. 5a). LPO production in BALF was also significantly lowered by edaravone injection on day $2(p=0.049$; fig. $5 b)$. The serum or BALF levels of LPO in edaravone-treated mice on day 7 after BLM challenge did not differ from those in untreated mice (data not shown).

\section{Effects of edaravone on the $P G E_{2}$ levels in BALF of the BLM model}

The $\mathrm{PGE}_{2}$ level in BALF was measured as an index of the amount of anti-inflammatory prostanoids. $\mathrm{PGE}_{2}$ was measured by immunoassay in BLM-treated mice with or without pretreatment of edaravone $\left(300 \mathrm{mg} \cdot \mathrm{kg}^{-1}\right.$ body weight). As shown in figure 6, mice pre-treated with edaravone exhibited significantly greater levels of $\mathrm{PGE}_{2}$ than mice receiving BLM alone on day 2 , but this elevation of $\mathrm{PGE}_{2}$ by edaravone rapidly decreased thereafter until day 7 (data not shown).

\section{Adverse effects of edaravone on the serum creatin levels in a model}

A temporary increase of serum creatinine levels was observed at the dose of $300 \mathrm{mg} \cdot \mathrm{kg}^{-1}$ of edaravone (fig. 7). However, the creatinine elevation at day 2 after BLM instillation was normalised until day 7 (fig. 7).

\section{DISCUSSION}

The present study has shown that the anti-inflammatory effects of edaravone improved the 28-day survival in mice with acute lung injury after a BLM instillation. Edaravone could mitigate the progression of lung injury and fibrosis. It also attenuated the cellular infiltration and the concentrations of LPO in BALF. These findings suggested that edaravone could inhibit lung 

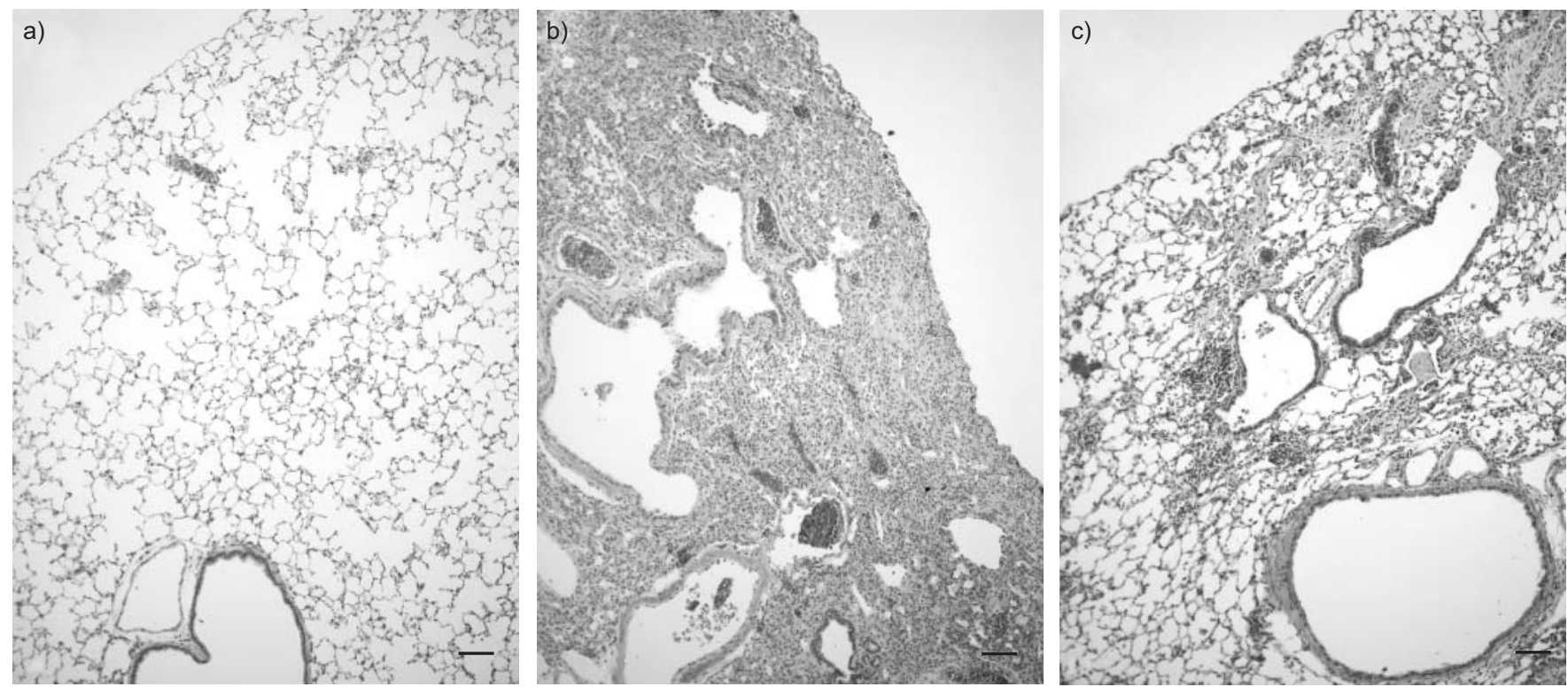

FIGURE 2. Effects of edaravone on histopathological changes. Lung tissue was obtained on day 28 after instillation of bleomycin (BLM) or saline and was stained with haematoxylin and eosin. a) Saline-group lung tissue sample showing thin interalveolar septa, a lack of inflamed cells, and normal-appearing bronchioles and alveolar ducts b) BLM-group lung tissue sample showing alveolitis and patchy fibrosis with destruction of the alveolar structure, mainly in the subpleural regions. c) In mice pre-treated with high doses of edaravone $\left(300 \mathrm{mg} \cdot \mathrm{kg}^{-1}\right)$ these features were less severe. Scale bars $=200 \mu \mathrm{m}$.

injury and fibrosis via the repression of LPO production in the current model.

In the present study, a murine BLM-induced pulmonary fibrosis model was used to examine the ability of edaravone to: 1) inhibit pulmonary fibrosis; 2) decrease lung inflammation and attenuate ROS. First, the ability of edaravone to inhibit pulmonary fibrosis was investigated using histological examination and

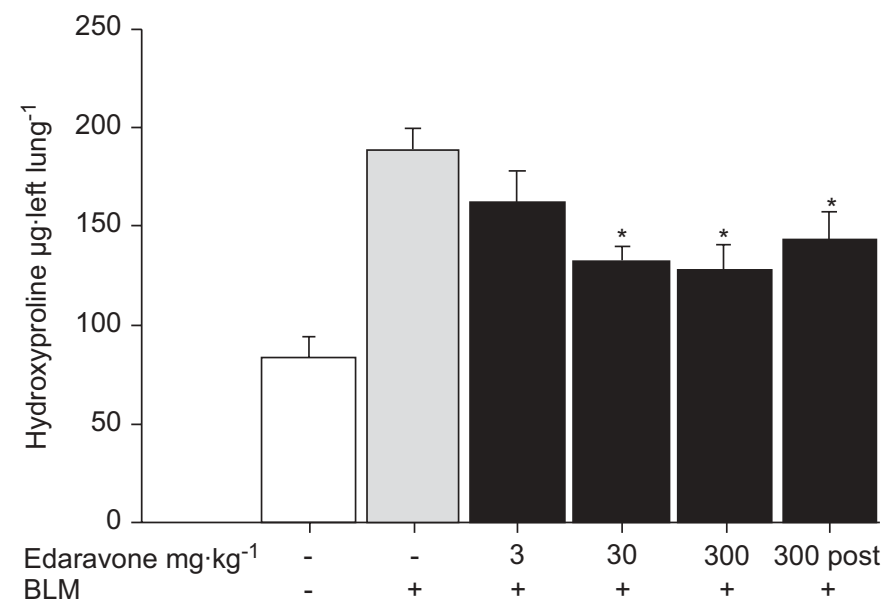

FIGURE 3. Effects of edaravone on the hydroxyproline content in the left lung in a bleomycin (BLM)-induced pulmonary fibrosis mouse model. The hydroxyproline content was significantly increased by BLM injection. Single administration of 30 or $300 \mathrm{mg} \cdot \mathrm{kg}^{-1}$ of edaravone $1 \mathrm{~h}$ before BLM instillation significantly attenuated the BLM-induced increase in hydroxyproline content on day 28. In addition, a single high dose (300 $\mathrm{mg} \cdot \mathrm{kg}^{-1}$ ) of edaravone by intraperitoneal infusion $24 \mathrm{~h}$ after the instillation of BLM also significantly decreased hydroxyproline contents. $\square$ : control group; I-: BLM group; $\mathbf{\square}:$ BLM + edaravone group. Data are presented as mean \pm SEM (six to 10 mice in each group). *: $p<0.05$ in comparison to the BLM group. measurement of hydroxyproline contents. It was found that a single administration of edaravone not only $1 \mathrm{~h}$ before but also $24 \mathrm{~h}$ after BLM challenge could mitigate the progression of pulmonary fibrosis on day 28 after BLM instillation.

Secondly, the ability of edaravone to decrease lung inflammation and attenuate ROS was investigated. The present study demonstrated that edaravone could attenuate the concentrations of LPO (an indicator of oxidative stress) in BALF and serum on day 2. An oxidant-antioxidant imbalance may contribute to the pathogenesis of BLM-induced pulmonary fibrosis [7-10]. HAGIWARA et al. [9] have shown that aerosolised administration of $\mathrm{N}$-acetylcysteine (NAC) attenuates lung fibrosis induced by BLM via repression of LPO production. In the present study, the number of total cells and neutrophils in BALF in edaravone-treated mice on day 7 was significantly decreased in comparison with untreated mice. These findings are consistent with previous reports [8-10]. Most of the antioxidant agents used for the treatment of BLM models have shown both antifibrosing effects and anti-inflammatory effects, i.e. attenuating the cellular infiltration, pro-inflammatory cytokines or chemokines in BALF [8-10]. Although pro-inflammatoy cytokines or chemokines in BALF were not measured, the current authors speculate that edaravone may have decreased the pro-inflammatory cytokine or chemokine production in the current BLM-induced lung injury model.

The present study demonstrated that a single administration of edaravone reduced the total hydroxyproline contents in BLMtreated lungs on day 28. Although numerous agents targeting diverse signalling and molecular pathways inhibited fibrosis very effectively in the BLM-induced pulmonary fibrosis model, so far none of the molecules have demonstrated clear efficacy in the treatment of IPF. One main difference between the disease and the mouse model is the inflammatory component 

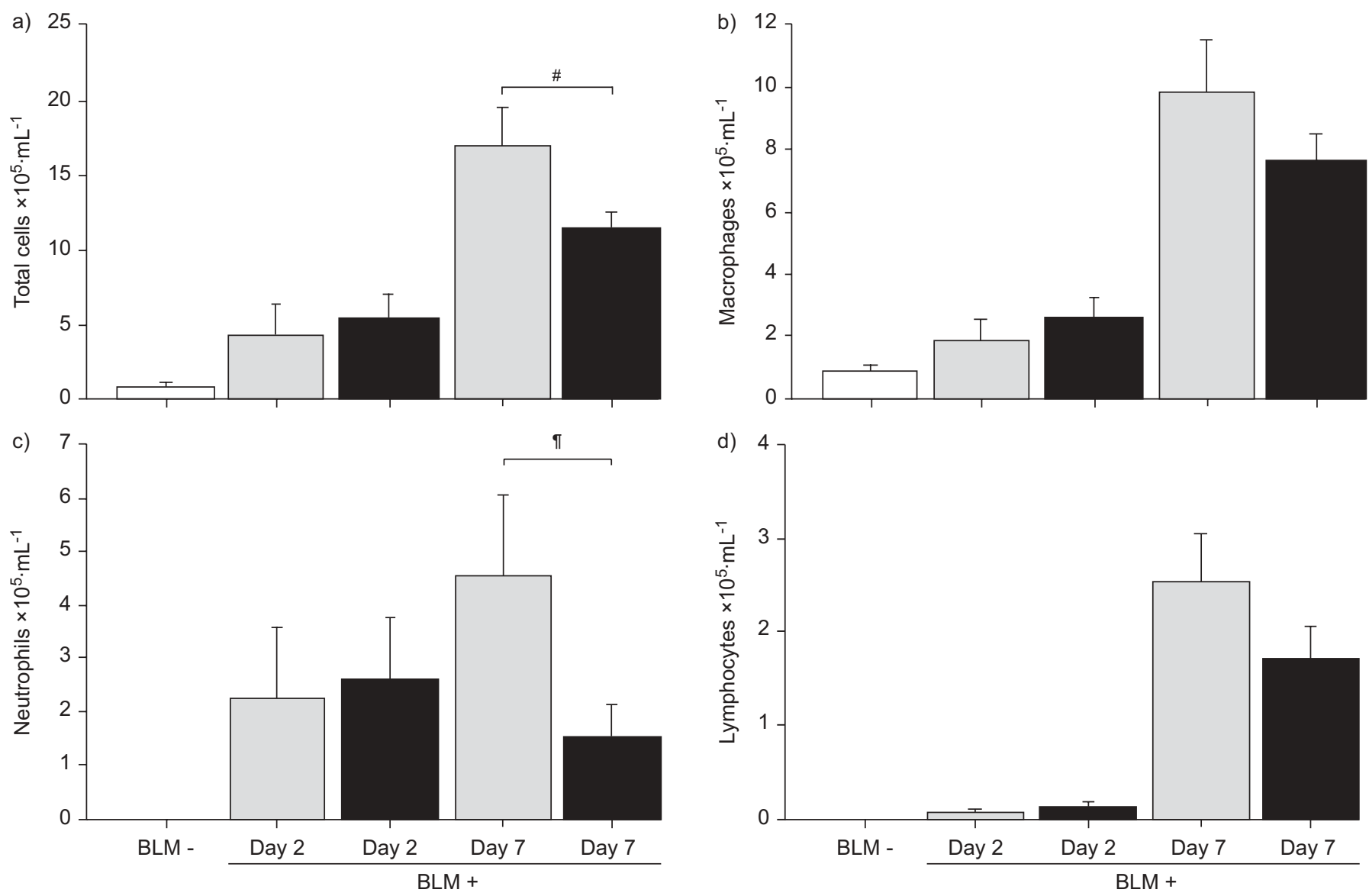

FIGURE 4. Effects of edaravone on bronchoalveolar lavage fluid (BALF) cell analysis in a bleomycin (BLM)-induced pulmonary fibrosis mouse model. Single administration of $300 \mathrm{mg} \cdot \mathrm{kg}^{-1}$ of edaravone $1 \mathrm{~h}$ before BLM instillation significantly reduced the number of total cells and neutrophils in BALF on day 7 ( $p<0.05$; a and $\mathrm{c}$ ). There was no change in the number of macrophages or lymphocytes in BALF on day 7 (b and d). $\square$ : control group; 1 : BLM group; $\mathbf{\square}:$ BLM + edaravone group. Data are presented as the mean \pm SEM ( $n=6$ in control and day 2 groups, $n=10$ in each day 7 group). ${ }^{*}: p=0.045 ;{ }^{\circ}: p=0.046$.

of early BLM-induced lung injury, which is often absent in human IPF [19]. Recently, CHAUDHARY et al. [20] determined the time-course of the development of inflammation and fibrosis in BLM-induced lung fibrosis. They demonstrated that in an animal model of single intratracheal injection of BLM, the "switch" between inflammation and fibrosis occurred on or just after day 9 [20]. Although the current authors experimented with daily intravenous or intraperitoneal injections of $60 \mathrm{mg} \cdot \mathrm{kg}^{-1}$ edaravone from 14 days after BLM instillation, there was no beneficial effect (data not shown). HAGIWARA et al. [9] used NAC inhalation and obtained results similar to those in the present study. The current results suggested that edaravone might not demonstrate a therapeutic effect on chronic fibrotic lung diseases, such as IPF, but may have a preventive effect in the very accelerated phases of interstitial lung diseases, such as in acute exacerbation of IPF, acute interstitial pneumonia or drug-induced lung diseases.

WATANABE et al. [21] have shown that edaravone acts as: 1) a radical scavenger; 2) a stimulator of PG production; 3) an inhibitor of lipoxygenease; and 4) a protector against cell membrane damage. Thus, it was considered that arachidonic acid might be preferentially metabolised via the alternative cyclooxygenase (COX) pathway to prostanoids that possess antiinflammatory and antifibrotic activity, e.g. $\mathrm{PGE}_{2} . \mathrm{PGE}_{2}$ is produced in large quantities by macrophages in response to pro-inflammatory molecules such as IL-1 and lipopolysaccharide [22-24] and is, therefore, also considered a pro-inflammatory mediator. In addition to its effects on inflammation, $\mathrm{PGE}_{2}$ suppresses fibroblast proliferation [25] and reduces collagen mRNA expression [26], thereby exerting an antifibrotic activity. In vivo, consistent with an antifibrotic activity of $\mathrm{PGE}_{2}, \mathrm{COX} 2$ knockout mice were found to be more susceptible to BLMinduced lung fibrosis [27]. The administration of edaravone before BLM challenge was found to produce more $\mathrm{PGE}_{2}$ in the BALF than saline administration. EGAN et al. [28] have shown that the COX-PG pathway is irreversibly self-deactivated due to the natural reduction of the hydroperoxide at carbon 15 of $\mathrm{PGG}_{2}$ to the hydroxyl on $\mathrm{PGH}_{2}$. During this reduction, radicals, possibly hydroxyl radicals, are formed and could oxidise the enzyme [28]. Therefore, edaravone may increase both the initial rate and the total reaction prior to deactivation by partially consuming these radicals. The current authors did not examine which cells (macrophages, epithelial cells, endothelial cells or fibroblasts) contribute to $\mathrm{PGE}_{2}$ production. Further examination will be needed to determine which cells are affected by edaravone. 

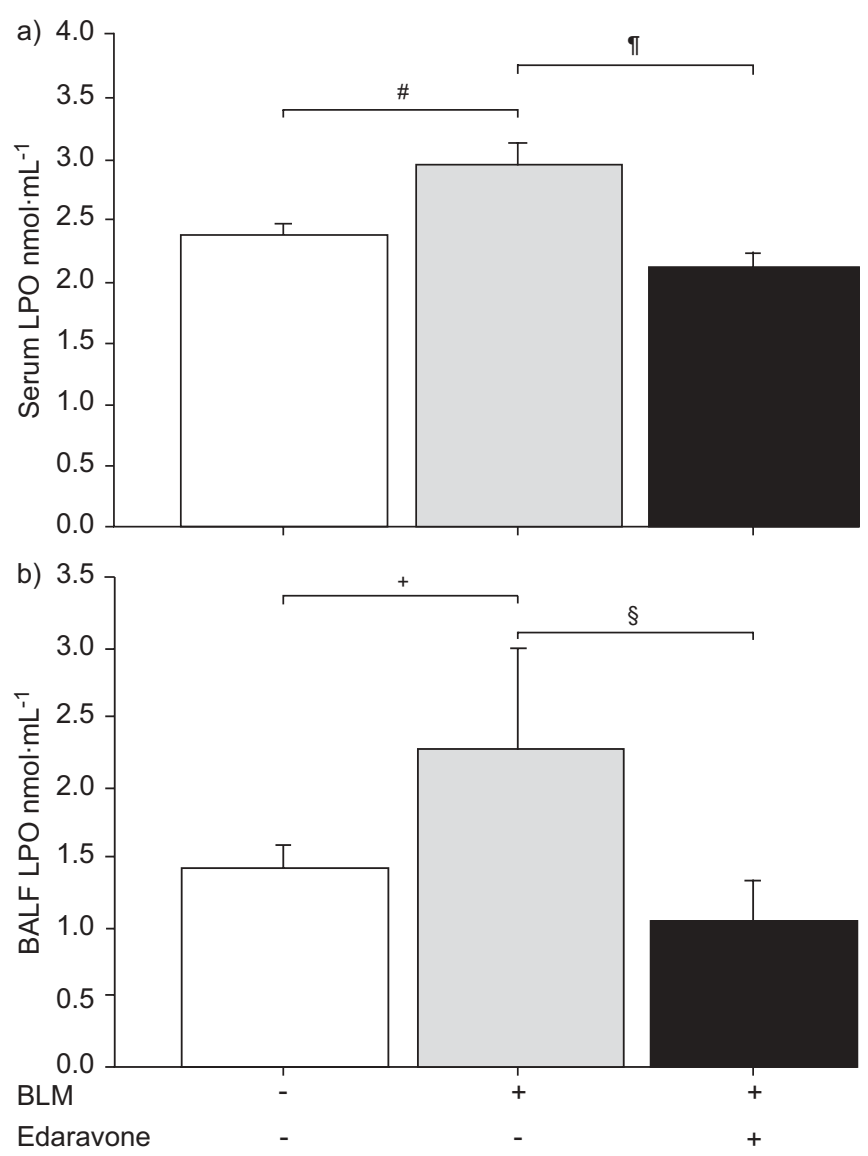

FIGURE 5. Effects of edaravone on the amount of a) lipid hydroperoxide (LPO) in serum and b) bronchoalveolar lavage fluid (BALF) in a bleomycin (BLM)-induced pulmonary fibrosis mouse model. Edaravone treatment consisted of a single administration of $300 \mathrm{mg} \cdot \mathrm{kg}^{-1} 1 \mathrm{~h}$ before BLM instillation. a) Although on day 2 after BLM instillation serum LPO levels were significantly increased compared with the control mice, administration of edaravone significantly decreased the levels of LPO in serum. b) LPO production in BALF was also significantly lowered by edaravone injection on day 2. $\square$ : control group; 1 : BLM group; $\mathbf{~ : ~ B L M ~ + ~ e d a r a v o n e ~ g r o u p . ~}$ Data are presented as the mean $\pm \operatorname{SEM}\left(n=6\right.$ in each group). ${ }^{*}: p=0.013$; $\because p=0.001 ;{ }^{+}: p=0.125 ;{ }^{s}: p=0.049$.

Usually, the daily dose of edaravone is $\sim 1.5 \mathrm{mg} \cdot \mathrm{kg}^{-1}$, and the treatment commences 14 days after cerebral infarction [11-14]. Although, in a previous report, no adverse effects on heart rate or blood pressure at the dose of $450 \mathrm{mg} \cdot \mathrm{kg}^{-1}$ of edaravone were reported [29], the present authors observed a temporary increase of serum creatinine levels at the dose of $300 \mathrm{mg} \cdot \mathrm{kg}^{-1}$ of edaravone. However, the creatinine elevation on day 2 after BLM instillation was normalised by day 7. No other adverse effects of a single daily administration of $300 \mathrm{mg} \cdot \mathrm{kg}^{-1}$ of edaravone were observed, despite the fact that this dose was $\sim 200$ times higher than the daily dose used in humans. ANZAI et al. [29] have reported a radioprotective effect of edaravone against whole body $\mathrm{X}$-ray irradiation in $\mathrm{C} 3 \mathrm{H}$ mice. To increase the survival rate, the necessary dose of edaravone was $450 \mathrm{mg} \cdot \mathrm{kg}^{-1}$ intraperitoneally, and the timing of the administration was $30 \mathrm{~min}$ prior to the irradiation [29]. AsAI et al. [16] used daily intravenous injections of $3 \mathrm{mg} \cdot \mathrm{kg}^{-1}$ edaravone for rabbits administered $2 \mathrm{mg} \cdot \mathrm{kg}^{-1}$ BLM. In the present study, a

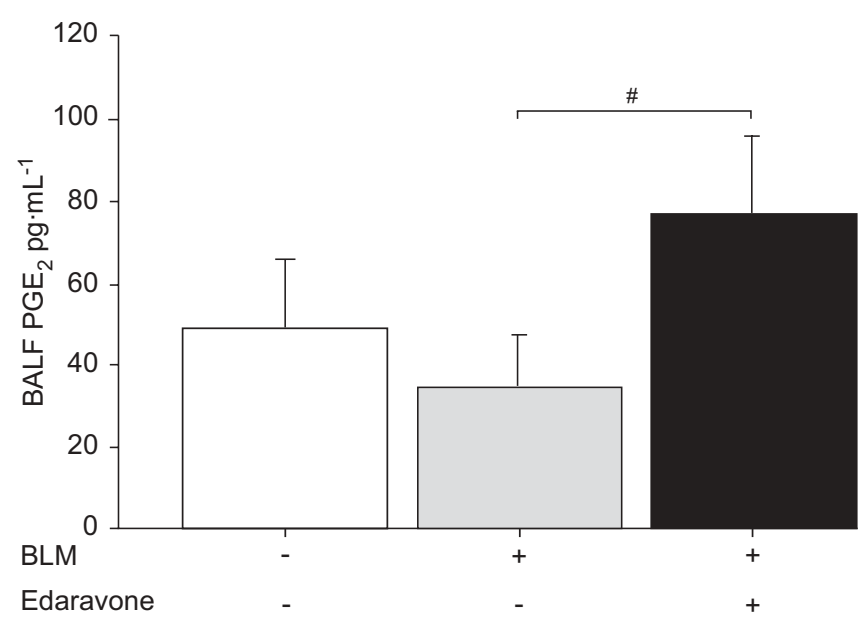

FIGURE 6. Effects of edaravone on the prostaglandin $(\mathrm{PG}) \mathrm{E}_{2}$ levels in bronchoalveolar lavage fluid (BALF) of a bleomycin (BLM)-induced pulmonary fibrosis mouse model. Single administration of $300 \mathrm{mg} \cdot \mathrm{kg}^{-1}$ of edaravone $1 \mathrm{~h}$ before BLM instillation significantly increased $\mathrm{PGE}_{2}$ on day 2. $\square$ : control group; 1 : BLM group; $\mathbf{~ : ~ B L M ~ + ~ e d a r a v o n e ~ g r o u p . ~ D a t a ~ a r e ~ p r e s e n t e d ~ a s ~ t h e ~ m e a n ~} \pm \mathrm{SEM}$ ( $n=6$ in each group). ${ }^{*}: p=0.043$.

high dose of edaravone was required for the treatment of lung injury in ICR mice. In addition to the dose-dependency, the efficacy of edaravone in ameliorating BLM-induced organ injury was also dependent on the administration route and the strain of mice.

In conclusion, the results of the present study suggest that edaravone could inhibit bleomycin-induced lung injury and fibrosis via the repression of lipid hydroperoxide production and augmentation of prostaglandin $\mathrm{E}_{2}$ production. Additional

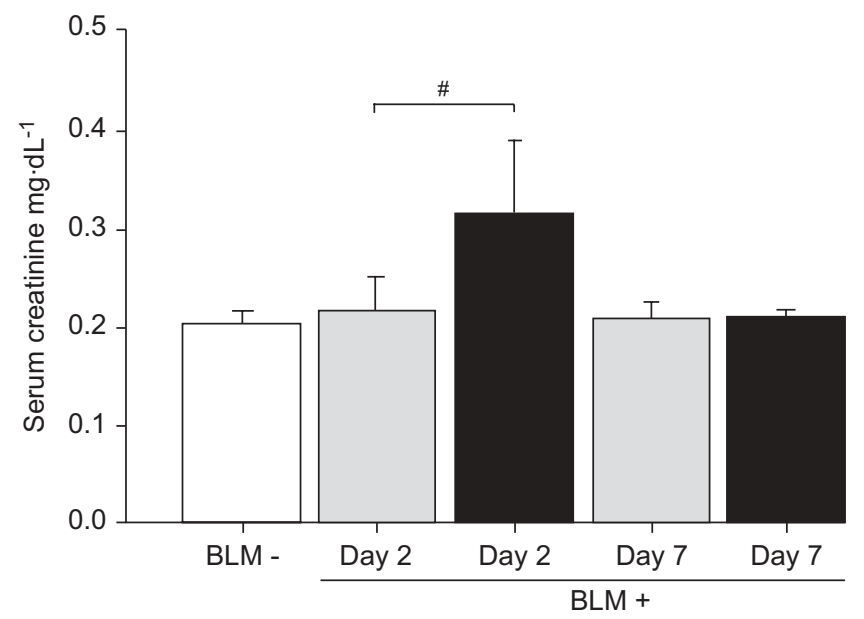

FIGURE 7. Adverse effects of edaravone on the serum creatinine levels in a bleomycin (BLM)-induced pulmonary fibrosis mouse model. The serum creatinine levels were measured by Mitsubishi Kagaku Bio-Clinical Laboratories, Inc. (Tokyo, Japan). Although a temporary increase of serum creatinine levels at the dose of $300 \mathrm{mg} \cdot \mathrm{kg}^{-1}$ of edaravone was observed on day 2 after BLM instillation, the elevation was normalised by day 7. $\square$ : control group; 1 : BLM group; $\mathbf{\square}$ : BLM + edaravone group. Data are presented as the mean \pm SEM ( $n=6$ in each group). \# : $p=0.044$. 
clinical studies on other fatal interstitial lung diseases, such as acute exacerbation of idiopathic pulmonary fibrosis, acute interstitial pneumonia associated with collagen vascular diseases or chemotherapy-related toxicity, are needed to determine the safest dose, administration route and duration times of edaravone.

\section{ACKNOWLEDGEMENTS}

The authors would like to thank T. Ikahata for (Division of Pulmonary Medicine, Dept of Medicine, Jichi Medical University) excellent assistance.

\section{REFERENCES}

1 American Thoracic Society. Idiopathic pulmonary fibrosis: diagnosis and treatment. International consensus statement. American Thoracic Society (ATS), and the European Respiratory Society (ERS). Am J Respir Crit Care Med 2000; 161: 646-664.

2 Fridovich I. The biology of oxygen radicals. Science 1978; 201: 875-880.

3 Freeman BA, Crapo JD. Biology of disease: free radicals and tissue injury. Lab Invest 1982; 47: 412-426.

4 Schraufstatter IU, Hyslop PA, Jackson J, Revak SD, Cochrane CC. Oxidant and protease injury of the lung. Bull Eur Physiopathol Respir 1987; 23: 297-302.

5 Demedts M, Behr J, Buhl R, et al. High-dose acetylcysteine in idiopathic pulmonary fibrosis. N Engl J Med 2005; 353: 2229-2242.

6 Sausville EA, Stein RW, Peisach J, Horwitz SB. Properties and products of the degradation of DNA by bleomycin and iron(II). Biochemistry 1978; 17: 2746-2754.

7 Galvan L, Huang CH, Prestayko AW, Stout JT, Evans JE, Crooke ST. Inhibition of bleomycin-induced DNA breakage by superoxide dismutase. Cancer Res 1981; 41: 5103-5106.

8 Cunningham ML, Ringrose PS, Lokesh BR. Inhibition of the genotoxicity of bleomycin by superoxide dismutase. Mutat Res 1984; 135: 199-202.

9 Hagiwara S, Ishii Y, Kitamura S. Aerosolized administration of $\mathrm{N}$-acetylcysteine attenuates lung fibrosis induced by bleomycin in mice. Am J Respir Crit Care Med 2000; 162: 225-231.

10 Tamagawa K, Taooka Y, Maeda A, Ohiyama K, Ishioka S, Yamakido M. Inhibitory effects of a lecithinized superoxide dismutase on bleomycin-induced pulmonary fibrosis in mice. Am J Respir Crit Care Med 2000; 161: 1279-1284.

11 Abe K, Yuki S, Kogure K. Strong attenuation of ischemic and postischemic brain edema in rats by a novel free radical scavenger. Stroke 1988; 19: 480-485.

12 Kawai H, Nakai H, Suga M, Yuki S, Watanabe T, Saito KI. Effects of a novel free radical scavenger, MCI-186, on ischemic brain damage in the rat distal middle cerebral artery occlusion model. J Pharmacol Exp Ther 1997; 281: 921-927.

13 Watanabe T, Yuki S, Egawa M, Nishi H. Protective effects of MCI-186 in cerebral ischemia: possible involvement of free radical scavenging and antioxidant actions. J Pharmacol Exp Ther 1994; 268: 1597-1604.
$14 \mathrm{Wu}$ TW, Zeng LH, Wu J, Fung KP. MCI-186: further histochemical and biochemical evidence of neuroprotection. Life Sci 2000; 67: 2387-2392.

15 Ito K, Ozasa H, Horikawa S. Edaravone protects against lung injury induced by intestinal ischemia/reperfusion in rat. Free Radic Biol Med 2005; 38: 369-374.

16 Asai T, Ohno Y, Minatoguchi S, et al. The specific free radical scavenger edaravone suppresses bleomycininduced acute pulmonary injury in rabbits. Clin Exp Pharmacol Physiol 2007; 34: 22-26.

17 Harrison JH, Lazo JS. High dose continuous infusion of bleomycin in mice: a new model for drug-induced pulmonary fibrosis. J Pharmacol Exp Ther 1987; 243: 1185-1194.

18 Stegman H, Stadler K. Determination of hydroxyproline. Clin Chim Acta 1967; 18: 267-273.

19 Selman M, King TE, Pardo A. Idiopathic pulmonary fibrosis: prevailing and evolving hypotheses about its pathogenesis and implications for therapy. Ann Intern Med 2001; 134: 136-151.

20 Chaudhary NI, Schnapp A, Park JE. Pharmacologic differentiation of inflammation and fibrosis in the rat bleomycin model. Am J Respir Crit Care Med 2006; 173: 769-776.

21 Watanabe T, Morita I, Nishi H, Murota S. Preventive effect of MCI-186 on 15-HPETE induced vascular endothelial cell injury in vitro. Prostaglandins Leukot Essent Fatty Acids 1988; 33: 81-87.

22 Mohr C, Davis GS, Graebner C, Hemenway DR, Gemsa D. Enhanced release of prostaglandin $\mathrm{E}_{2}$ from macrophages of rats with silicosis. Am J Respir Cell Mol Biol 1992; 6: 390-396.

23 Watson J, Wijelath ES. Interleukin-1 induced arachidonic acid turnover in macrophages. Autoimmunity 1990; 8: 71-76.

24 Tilley SL, Coffman TM, Koller BH. Mixed messages: modulation of inflammation and immune responses by prostaglandins and thromboxanes. J Clin Invest 2001; 108: 15-23.

25 Elias JA, Rossman MD, Zurier RB, Daniele RP. Human alveolar macrophage inhibition of lung fibroblast growth. A prostaglandin-dependent process. Am Rev Respir Dis 1985; 131: 94-99.

26 Clark JG, Kostal KM, Marino BA. Modulation of collagen production following bleomycin-induced pulmonary fibrosis in hamsters: presence of a factor in lung that increases fibroblast prostaglandin $E_{2}$ and cAMP and suppresses fibroblast proliferation and collagen production. J Biol Chem 1982; 257: 8098-8105.

27 Keerthisingam CB, Jenkins RG, Harrison NK, et al. Cyclooxygenase-2 deficiency results in a loss of the antiproliferative response to transforming growth factor- $\beta$ in human fibrotic lung fibroblasts and promotes bleomycininduced pulmonary fibrosis in mice. Am J Pathol 2001; 158: 1411-1422.

28 Egan RW, Paxton J, Kuehl FA Jr. Mechanism for irreversible self-deactivation of prostaglandin synthetase. J Biol Chem 1976; 251: 7329-7335.

29 Anzai K, Furuse M, Yoshida A, et al. In vivo radioprotection of mice by 3-methyl-1-phenyl-2-pyrazolin-5-one (edaravone; Radicut), a clinical drug. J Radiat Res (Tokyo) 2004; 45: 319-323. 